\title{
The Large UV/Optical/Infrared Surveyor (LUVOIR): Decadal Mission Study Update
}

Matthew R. Bolcar*a, Julie Crooke ${ }^{a}$, Jason E. Hylan ${ }^{\mathrm{a}}$, Ginger Bronke ${ }^{\mathrm{a}}$, Christine Collins ${ }^{\mathrm{a}}$, James Corsetti $^{\mathrm{a}}$, Joe Generie ${ }^{\mathrm{a}}$, Qian Gong ${ }^{\mathrm{a}}$, Tyler Groff ${ }^{\mathrm{a}}$, William Hayden ${ }^{\mathrm{a}}$, Andrew Jones ${ }^{\mathrm{a}}$, Bryan

Matonak $^{\mathrm{a}}$, Sang Park ${ }^{\mathrm{b}}$, Lia Sacks ${ }^{\mathrm{a}}$, Garrett West ${ }^{\mathrm{a}}$, Kan Yang ${ }^{\mathrm{a}}$, Neil Zimmerman ${ }^{\mathrm{a}}$

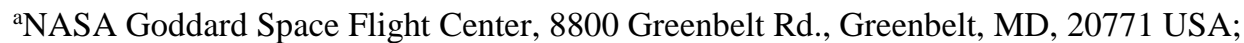

bHarvard Smithsonian Center for Astrophysics, 60 Garden St., Cambridge, MA 02138 USA

\begin{abstract}
NASA commissioned the study of four large mission concepts, including the Large Ultraviolet / Optical / Infrared (LUVOIR) Surveyor, to be evaluated by the 2020 Decadal Survey in Astrophysics. In response, the Science and Technology Definition Team (STDT) identified a broad range of science objectives for LUVOIR that include the direct imaging and spectral characterization of habitable exoplanets around sun-like stars, the study of galaxy formation and evolution, the exchange of matter between galaxies, star and planet formation, and the remote sensing of Solar System objects. To meet these objectives, the LUVOIR Study Office, located at NASA's Goddard Space Flight Center (GSFC), completed the first design iteration of a 15-m segmented-aperture observatory that would be launched by the Space Launch System (SLS) Block 2 configuration. The observatory includes four serviceable instruments: the Extreme Coronagraph for Living Planetary Systems (ECLIPS), an optical / near-infrared coronagraph capable of delivering $10^{-10}$ contrast at inner working angles as small as $2 \lambda / \mathrm{D}$; the LUVOIR UV Multi-object Spectrograph (LUMOS), which will provide low- and medium-resolution UV (100 - $400 \mathrm{~nm})$ multi-object imaging spectroscopy in addition to far-UV imaging; the High Definition Imager (HDI), a high-resolution wide-field-of-view NUV-Optical-NIR imager; and Pollux, a high-resolution UV spectro-polarimeter being contributed by Centre National d'Etudes Spatiales (CNES). The study team has executed a second design iteration to further improve upon the 15-m concept, while simultaneously studying an 8-m concept. In these proceedings, we provide an update on these two architectures.
\end{abstract}

Keywords: space telescopes, ultraviolet, optical, infrared, coronagraphy, ultra-stable systems

\section{INTRODUCTION}

The Astrophysics Division of NASA's Science Mission Directorate commissioned the study of four large mission concepts in preparation for the 2020 Decadal Study ${ }^{1}$ : the Large UV/Optical/Infrared Surveyor (LUVOIR) ${ }^{2}$, the Habitable Exoplanet Imager $(\mathrm{HabEx})^{3}$, the Origins Space Telescope (OST, formerly the Far-Infrared Surveyor) ${ }^{4}$, and Lynx (formerly the Xray Surveyor) ${ }^{5}$. Each of the studies is led by a Science and Technology Definition Team (STDT) tasked with defining a compelling science case and guiding the design of mission architectures commensurate with that science case. The LUVOIR STDT and Study Office have worked to develop two mission concepts capable of achieving the science objectives identified by the STDT.

The paper is organized as follows: The remainder of Section 1 briefly outlines the LUVOIR science case, and discusses the design philosophy adopted by the LUVOIR STDT and Study Office. Sections 2 and 3 provide updates on the LUVOIRA and LUVOIR-B designs, respectively. Section 4 provides details on the instruments being studied for both architectures, and Section 5 concludes the paper and describes future work.

\subsection{Science with LUVOIR}

LUVOIR's STDT has developed a breadth of compelling science cases that appeals to a broad section of the astrophysics community with three main themes: cosmic origins, exoplanets, and the Solar System.

The cosmic origins science case is defined by the ability to deliver a breadth of observational capabilities at unprecedented sensitivity and resolution. As demonstrated by the Hubble Space Telescope (HST), a capable, flexible, and upgradeable observatory allows us to answer the scientific questions of tomorrow that we do not even know to ask today. These questions range in topic and in scale from "How do galaxies evolve?" to "What are the building blocks of cosmic

*matthew.bolcar@nasa.gov; phone 1301 286-5237; fax 1301 286-0204; www.nasa.gov/goddard 
structure?" to "How do stars and planets form?" 6 Fundamentally, these questions regarding cosmic origins are of the nature, "How did we come to be?"

The exoplanet science case seeks to answer the questions, “Are we alone?”, and “Are we unique?”, requiring the ability to directly image habitable exoplanets around sun-like stars, and spectroscopically characterize their atmospheres. Thus, LUVOIR does not just seek to determine if life is present elsewhere, but also how common life might be throughout the galaxy, constraining the frequency of habitable (or perhaps, inhabited) planets through a statistical survey of hundreds of planetary systems. During this search, hundreds of other planets would be observed and characterized, allowing for a complete census of the "exoplanet zoo." Figure 1 shows a simulated Earth-analog spectrum as it would be detected by the 15-m LUVOIR-A architecture. The simulation was performed using LUVOIR’s online simulation tools?.

\section{Earth twin at 5 pc with LUVOIR-A, 50 hours per coronagraphic bandpass}

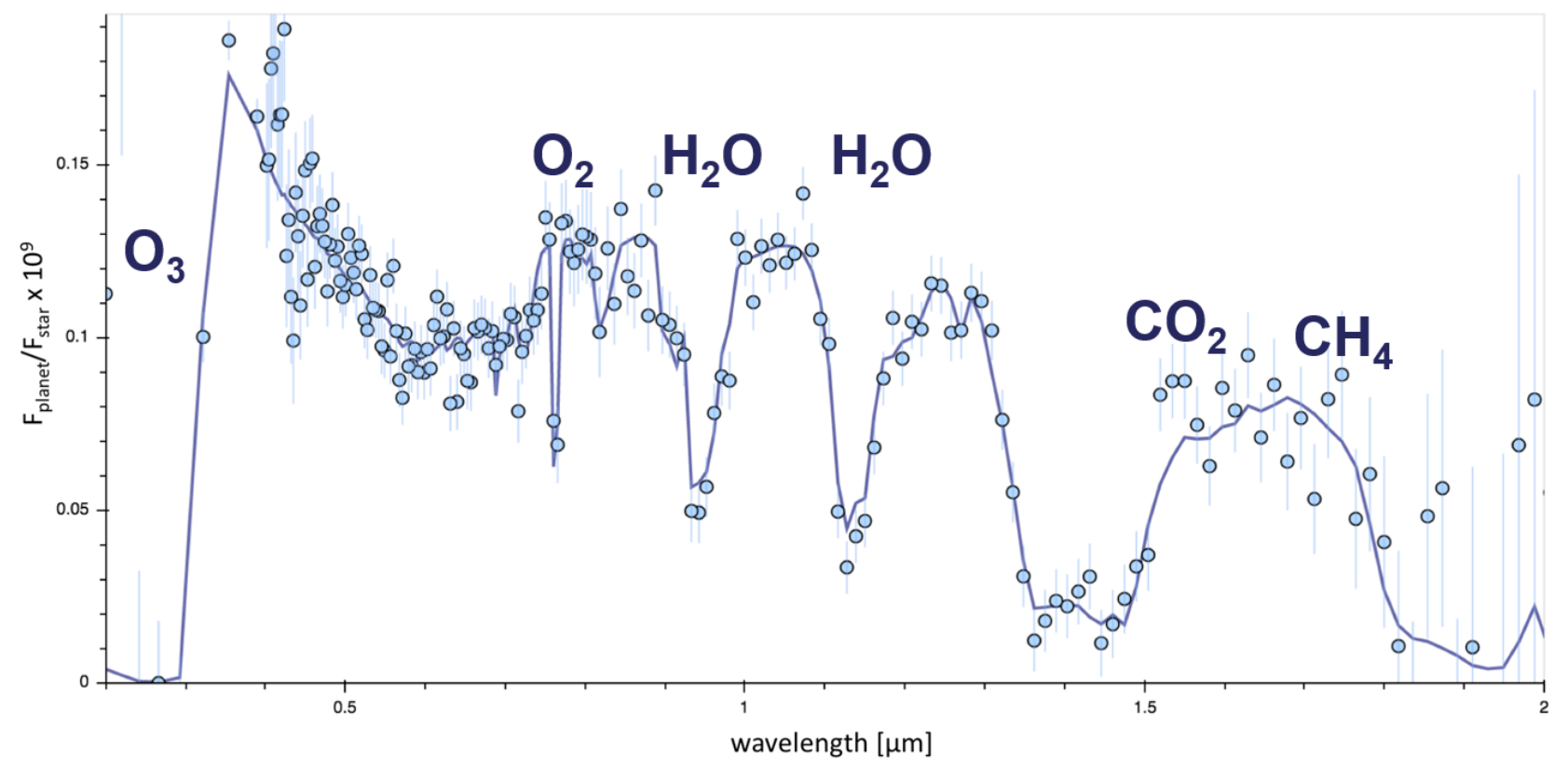

Figure 1 - A simulated direct spectrum of the modern earth orbiting a Sun-like star at 5 pc, as observed by the LUVOIR-A observatory. Key spectral features indicative of habitability are labelled.

The same capabilities that enable the cosmic origins and exoplanet science would also enable groundbreaking observations within our own solar system with resolutions previously achievable only by planetary probe missions. LUVOIR would turn these once-in-a-lifetime science campaigns into routine studies, while simultaneously enabling new observations of comets, asteroids, and Kuiper belt objects.

These compelling science objectives define a set of high-level mission capabilities: sensitivity, resolution, flexibility, highcontrast imaging, and mission duration. The LUVOIR Study team has used these capabilities to define a set of fundamental requirements that include: a large (8-15 meter) aperture; broad wavelength sensitivity ( 100 nm - 2.0 $\mu \mathrm{m})$; a suite of upgradeable instruments with imaging, spectroscopic, and high-contrast capabilities; and a long mission lifetime enabled through serviceability.

At the same time, the science objectives, and specifically the high-contrast imaging capability, drive the most difficult technical challenges of the LUVOIR concepts: picometer-level wavefront stability; large, low-noise, high-sensitivity focal planes; and highly reflective, uniform mirror coatings. A number of technology development efforts are underway to address these challenges, although they are beyond the scope of this paper. For more details on technology development the reader is referred to Ref. 6. 


\subsection{A Tale of Two LUVOIRs}

The LUVOIR Study Team has chosen to study two architectures in detail: LUVOIR-A and LUVOIR-B. These two architectures bracket a range of aperture diameters (15-m and 8-m, respectively), scientific capability, cost, and risk. This approach provides an evaluation of the science yield as a function of cost and risk, and allows the Decadal Survey to prioritize a compelling science mission when faced with a number of uncertainties such as: the future budget scenario; the availability of certain launch vehicles; and the as yet unknown discoveries of both the James Webb Space Telescope (JWST) and the Wide-field Infrared Survey Telescope (WFIRST).

A key aspect of the LUVOIR-B architecture was that it should fit into a "conventional” heavy-lift launch vehicle, whereas LUVOIR-A was designed specifically to use the full capabilities of NASA's planned Space Launch System (SLS) Block 2 vehicle with an 8.4-m x 27.4-m fairing. The LUVOIR-B architecture would reduce overall mission cost and risk should the SLS Block 2 vehicle be developed too late to be used by LUVOIR, or not achieve the current expected performance. With the word "conventional", the team implied a launch vehicle similar to the existing United Launch Alliance Delta IVHeavy vehicle with a 5-m x 19.8-m fairing, and a lift capability to the second Sun-Earth Lagrange point (SEL2) of 10,000 $\mathrm{kg}$. As described in Section 3, this definition eventually changed to be just a 5-m-class fairing as even the largest of the expected non-SLS heavy lift vehicles still do not have the required mass capability.

\section{LUVOIR-A STATUS UPDATE}

LUVOIR-A is the larger of the two architectures, with a 15-m diameter deployable, obscured segmented aperture, and includes a suite of four instruments:

- The Extreme Coronagraph for Living Planetary Systems (ECLIPS): a near-ultraviolet (UV) to near-infrared (NIR) coronagraph for high-contrast imaging and spectroscopy;

- The High Definition Imager (HDI): a near-UV to NIR wide-field of view imager;

- The LUVOIR UV Multi-Object Spectrograph (LUMOS): a far-UV to near-UV multi-object spectrograph, with far-UV imaging capabilities; and

- Pollux: a far-UV to near-UV high-resolution spectropolarimeter.

The Pollux instrument is being studied by a consortium of European partners, led by the Centre National d'Etudes Spatiales (CNES). For more information on Pollux, the reader is referred to Refs. 8 and 9. The other three instruments are described in more detail in Section 4 and in Ref. 6.

A full mission design of LUVOIR-A was completed towards the end of the summer of 2017, at which point initial analyses of the science yield were completed. While the overall system had excellent performance for both the cosmic origins and solar system science objectives, the exoplanet science - and specifically the expected yield of habitable exoEarths - was substantially lower than expected. Earlier studies ${ }^{10}$ implied that for a $15-\mathrm{m}$ aperture, one would expect $>60$ exoEarths. Instead, the first iteration of LUVOIR-A was yielding fewer than 10 exoEarths.

After evaluating the yield, it was determined that numerous design decisions contributed to this low exoEarth yield, including but not limited to:

1. A large central obscuration of the primary mirror.

The initial design of the optical telescope assembly (OTA) used a three-mirror anastigmat optical design to correct the wavefront error over a wide field of view. This design required a large hole in the primary mirror to pass the ray bundle to the aft-optics system. The segmentation geometry resulted in a larger hole than was strictly required by the ray bundle, resulting in an obscuration ratio (circumscribing obscuration diameter / inscribing primary mirror diameter) of $\sim 27 \%$. This obscuration ratio substantially suppressed the performance of the coronagraphs designed to work for LUVOIR-A.

2. A $>50 \%$ throughput loss in the coronagraph spectrometer.

The first design of ECLIPS used fiber-fed spectrometers as the back-end spectroscopic instruments, as opposed to more conventional integral field spectrographs. The fiber fed spectrometers were chosen to implement new techniques in high-resolution template matching spectroscopy ${ }^{11}$. While promising, these techniques require a 50/50 beamsplitter 
in the beam path such that the fiber can be aligned to the target planet, contributing to a $50 \%$ throughput loss after accounting for all of the reflectivity losses in the system. Additional fiber-coupling inefficiencies further reduced the coronagraph throughput.

3. A large number of low-reflectivity coatings required to support the UV channel of the coronagraph.

As mentioned earlier, the LUVOIR-A OTA uses a three mirror anastigmat design, and has a fourth fast-steering mirror at the real exit pupil for fine pointing control. These four mirrors use a protected aluminum coating to provide highthroughput for LUVOIR's far-UV science case. An additional four mirrors were required to pre-condition and fold the beam from the OTA into the coronagraph channels. These mirrors also required a protected aluminum coating in order to support the near-UV channel of the coronagraph. Thus, there were eight protected aluminum mirrors prior to the visible channel of the coronagraph (where exoplanet detections will occur), substantially reducing the instrument throughput in the visible band.

4. Performing spectral characterization of the $\sim 940 \mathrm{~nm}$ water line with a noisier detector.

The three channels in the first version of the coronagraph instrument were divided such that the transition between the visible and NIR channels occurred at $850 \mathrm{~nm}$. This meant that the $940 \mathrm{~nm}$ water spectral feature would be detected in the NIR channel with a Hawaii-4RG HgCdTe detector. While these detectors have seen substantial development in recent years, they still exhibit several electrons of read noise, requiring much longer integration times to guarantee a high-enough signal-to-noise ratio (SNR) detection. These longer integration times reduced the total number of observations that are possible in limited exposure time and, therefore, the exoEarth yield.

5. An unclear and non-optimal concept of operations.

The initial concept of operations envisioned using two of the three channels (visible and NIR) to execute the exoplanet detection search, while the third channel (near-UV) would be used for out-of-band wavefront sensing. Additionally, what would count as an "exoplanet detection" was poorly defined: would it require color information? Planet orbits? A water detection? Or a complete spectral characterization? This lack of clarity made it difficult to define a search strategy and thus optimize the exoEarth yields.

Given these issues, the LUVOIR STDT decided to execute a redesign effort of LUVOIR-A, specifically to optimize the system for better exoEarth yields while maintaining the capability of the observatory for cosmic origins and solar system science. The study team rapidly completed a number of trades and made the following changes to address the above issues:

1. Redesigned the OTA optical prescription and segment geometry to reduce the central obscuration.

A new three-mirror anastigmat design was developed that allowed for a much tighter ray bundle to be passed to the aft-optics system. When combined with a change in the segment size and segmentation geometry, this allowed the obscuration ratio to be reduced from $\sim 27 \%$ to $10 \%$, vastly improving the coronagraph performance. Figure 2 shows a comparison of the aperture geometries, while Figure 3 shows a comparison of the optical designs. 


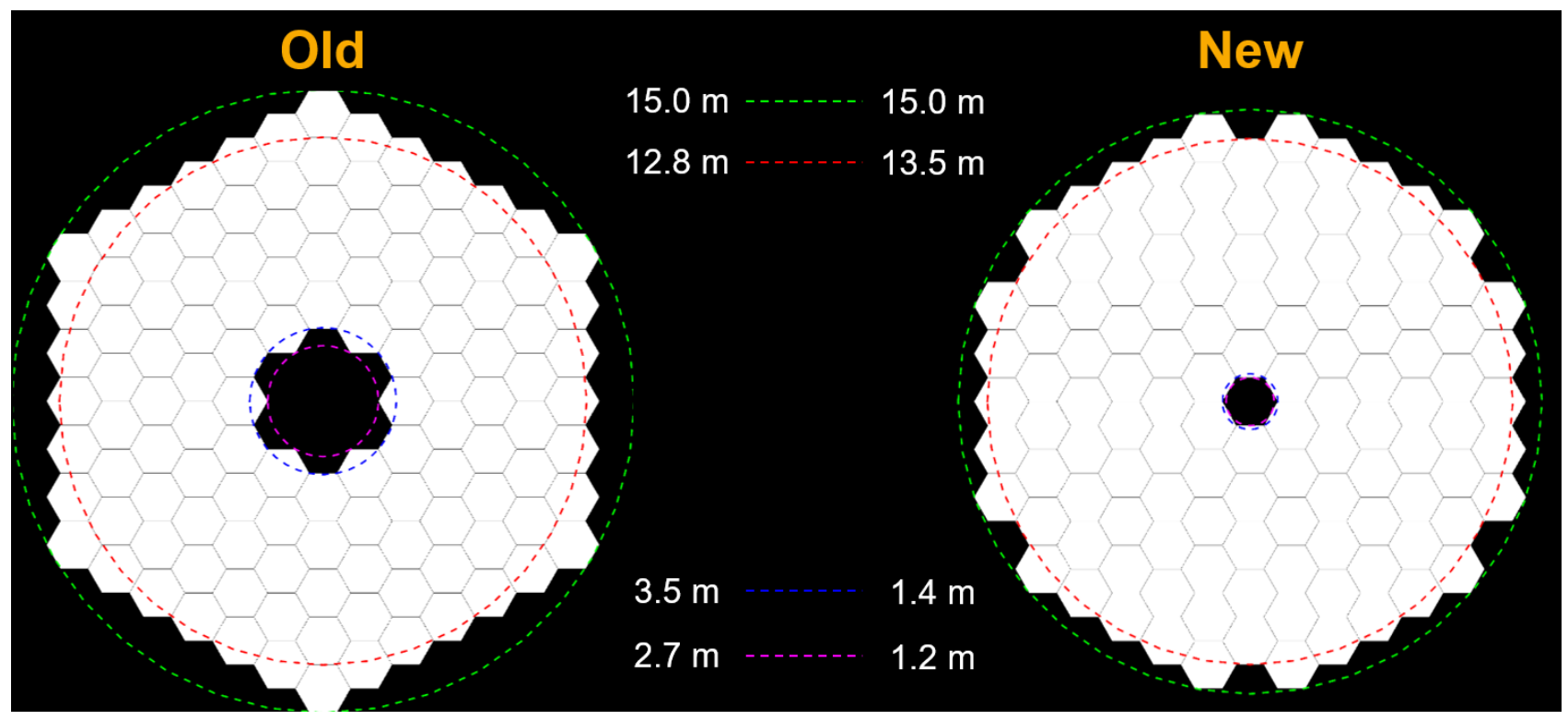

Figure 2 - Comparison of the old LUVOIR-A aperture geometry (Left) with new, redesigned LUVOIR-A (Right). To key differences that improved the coronagraph performance were the reduction of the central obscuration (from a $27 \%$ obscuration to a $10 \%$ obscuration), and an increase in the inscribing diameter from $12.8 \mathrm{~m}$ to $13.5 \mathrm{~m}$ - all while maintaining the outer diameter of $15.0 \mathrm{~m}$.
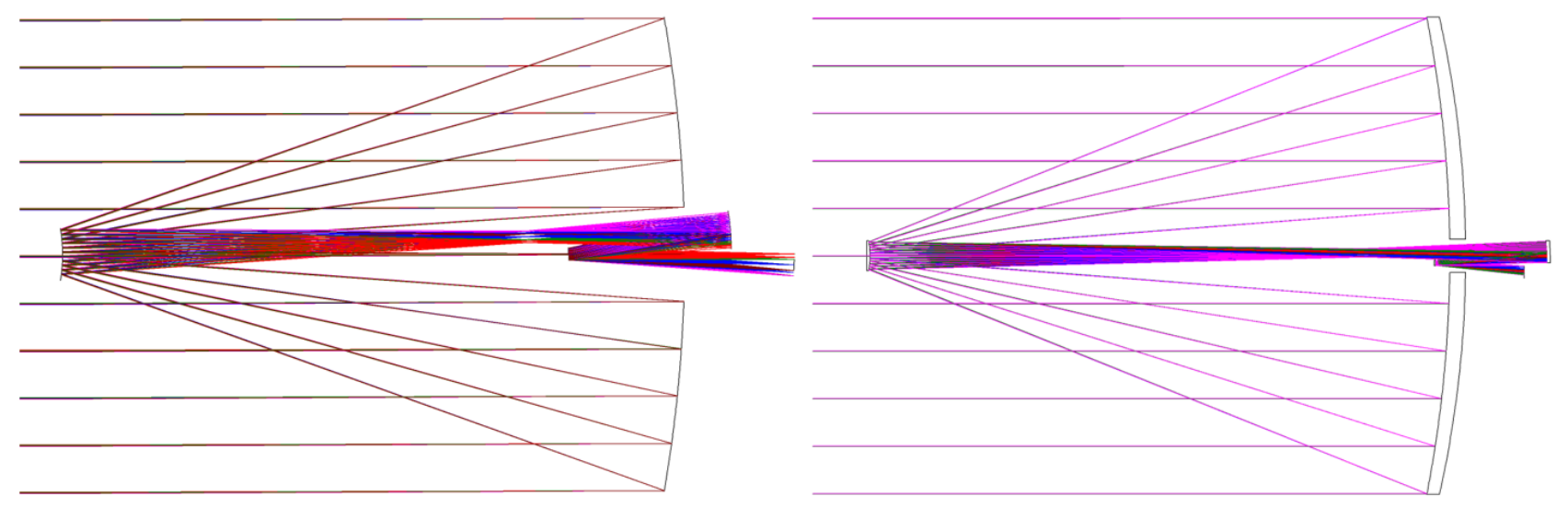

Figure 3 - Comparison of the old LUVOIR-A optical design (Left), with the new, redesigned LUVOIR-A (Right). Note the considerable reduction in the size of the hole required in the primary mirror for access to the aft-optics system. Another benefit of the new design is the location of the fast steering mirror inside the sag of the primary mirror such that it no longer needs to be deployed on orbit.

2. Implemented an integral field spectrograph in the coronagraph instrument visible and NIR channels.

Both the visible and NIR channels were redesigned to include an integral field spectrograph back-end instrument that can be accessed via a flip-in mirror, instead of a 50/50 beamsplitter. This design choice improved detection and characterization throughputs, as well as allowed for multiplexing spectral characterization, improving both detection yields of not only exoEarths, but other planets as well.

3. Redesigned the optical train between the telescope and the coronagraph.

After redesigning the OTE, the study team was able to remove one of the four reflections that pre-conditioned the beam and directed it into the coronagraph instrument, yielding a small improvement in throughput.

4. Re-optimized the spectral coverage of each channel within the coronagraph instrument. 


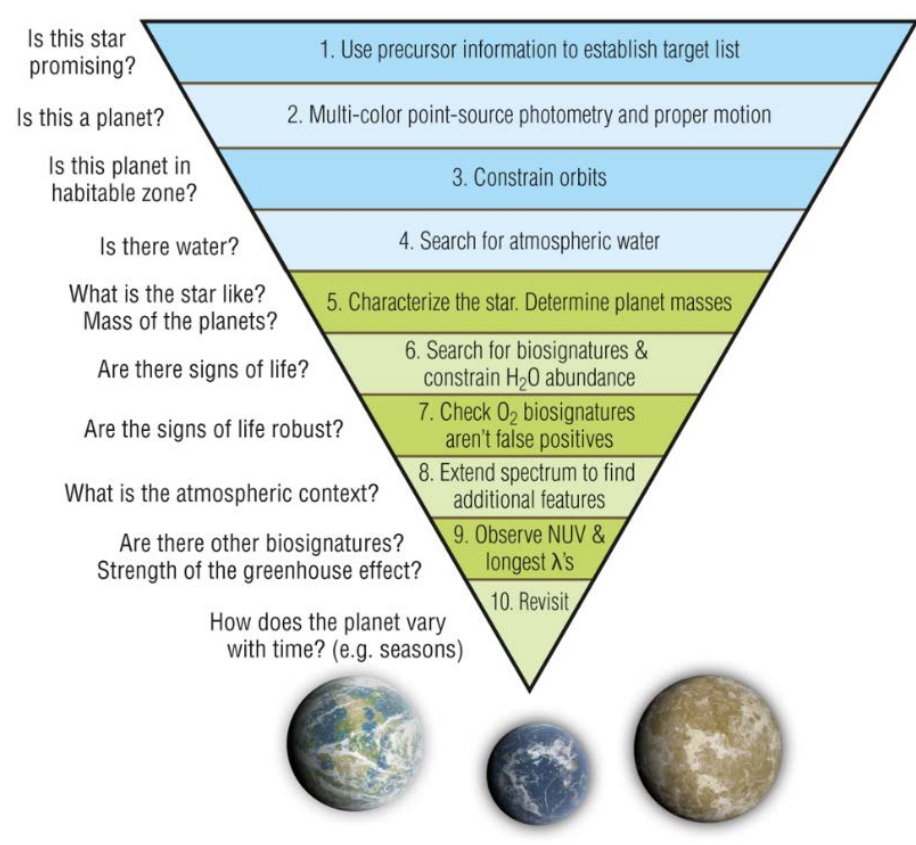

Figure 4 - Concept of Operations for executing the exoEarth search and characterization study. If the answer to the top four questions is "yes", then an exoEarth candidate has been detected, and additional characterization will be performed to determine habitability.
By shifting the transition between the visible and NIR channels within the coronagraph instrument to occur at $1000 \mathrm{~nm}$, the water detection can occur in the visible channel on a red-optimized electron multiplying CCD (EMCCD) detector. These detectors have an effective read noise of 0 , thus substantially reducing the integration time needed to achieve adequate signal-to-noise ratio.

5. Developed an optimized concept of operations.

In addition to allowing all three channels of the instrument to be used for detection, the exoplanet science team developed a concept of operations that clearly defines the steps necessary to identify an exoEarth candidate. This process is shown in Figure 4.

After completing these redesigns the exoEarth yield of the LUVOIR-A architecture is now estimated to be 50 exoEarths, exceeding the LUVOIR STDT's goal of achieving 30 exoEarth detections. The cosmic origins and solar system science performance actually improved, due to a larger total collecting area with the new aperture geometry.

Figure 5 shows the LUVOIR-A observatory concept. The primary mirror is composed of 120 segments that are 1.223-m flat-to-flat. Many of the other design features from the original LUVOIR-A architecture remain unchanged, and we refer the reader to Refs. 6 and 12 for additional details. 


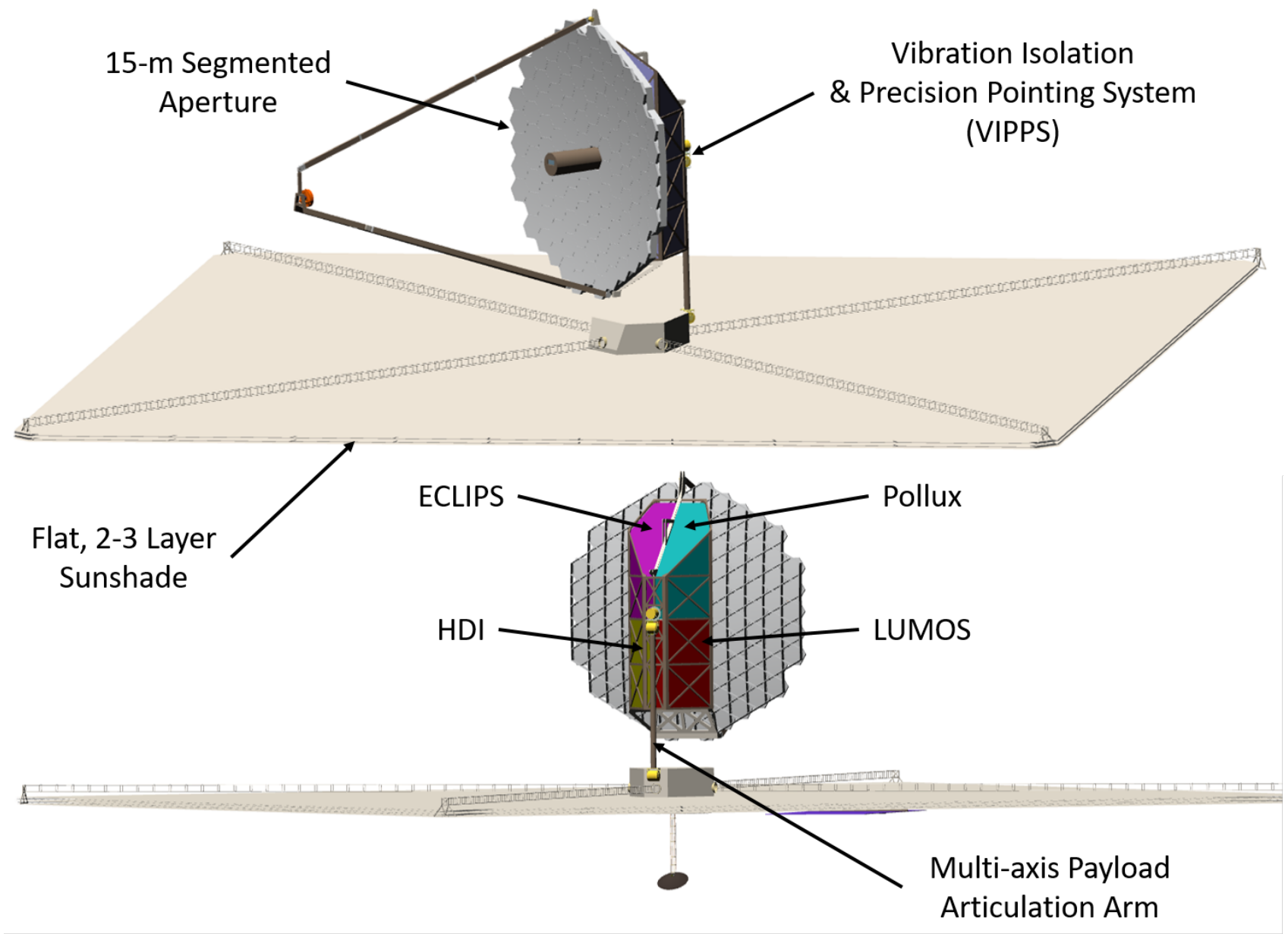

Figure 5 - The LUVOIR-A observatory concept.

\section{LUVOIR-B STATUS UPDATE}

LUVOIR-B was originally planned to be a scaled-down version of LUVOIR-A, with a 9-m segmented aperture and a similar optical design and instrument suite. However, the exoEarth yield exercise on LUVOIR-A called into question whether this plan for LUVOIR-B was still viable. Previous studies ${ }^{10}$ have shown that the exoEarth yield scales approximately as the square of the diameter of the aperture $\left(\mathrm{D}^{2}\right)$. With the LUVOIR-A $15-\mathrm{m}$ aperture estimated to find 50 exoEarths, one would expect a comparable 9-m aperture to find $\sim 18$. This is well below the goal of 30 exoEarths to constrain the frequency of habitable planets to less than $10 \%$ with $95 \%$ confidence. Thus, the LUVOIR study team decided to execute a second set of trades targeted at determining the LUVOIR-B architecture. Four questions guided the trade study:

1. What is the expected exoEarth yield of a 9-m obscured, segmented aperture system? This question addresses the original plan for LUVOIR-B being a scaled down version of LUVOIR-A.

2. What size aperture is necessary to achieve the minimum science requirement of 30 exoEarths?

3. What size aperture is necessary to achieve the minimum science requirement of 30 exoEarths, if additional science performance is sacrificed? This question addresses the fact that certain characteristics of the LUVOIR design reduce the exoEarth yield in return for specific observation capabilities. One example of this is the UV channel in the coronagraph: by removing that channel, the pre-optics can be coated in silver, improving the total optical throughput by $\sim 13 \%$ and improving exoEarth yield, but at the cost of not being able to discover and characterize Archean Earth candidates. 
4. Is it feasible to package and deploy an off-axis, unobscured segmented aperture from a 5-m-class fairing? If so, how large an aperture can be stowed, and what is the expected yield of that aperture? These questions address the fact that current coronagraph architectures, having benefited from years of development, work better with unobscured apertures. However, packaging constraints initially led the team to believe that a sufficiently large off-axis system could not be stowed in a 5-m fairing.

For each of these questions, a second question also needed to be answered: What launch vehicle capabilities would be required to launch the specified system? Table 1 summarizes the findings of this trade study.

Table 1 - Summary of results from LUVOIR-B Architecture Trade Study.

\begin{tabular}{|c|c|c|c|}
\hline $\begin{array}{c}\text { Aperture Diameter } \\
\text { / Type }\end{array}$ & $\begin{array}{c}\text { Estimated } \\
\text { exoEarth Yield }\end{array}$ & $\begin{array}{c}\text { Required Fairing } \\
\text { Diameter }\end{array}$ & $\begin{array}{c}\text { Required Lift } \\
\text { Capability }\end{array}$ \\
\hline 9-m Obscured & 16 & $5-\mathrm{m}$ & $>14,000 \mathrm{~kg}$ \\
\hline 11.6-m Obscured & 30 & $>5-\mathrm{m}$ & $>18,500 \mathrm{~kg}$ \\
\hline $\begin{array}{c}10.7-\mathrm{m} \text { Obscured } \\
\text { (w/ Reduced } \\
\text { Science Capability) }\end{array}$ & 30 & $>5-\mathrm{m}$ & $>16,700 \mathrm{~kg}$ \\
\hline 8-m Unobscured & 28 & $5-\mathrm{m}$ & $>14,000 \mathrm{~kg}$ \\
\hline
\end{tabular}

As the table shows, none of these options is capable of being launched by a Delta IV-Heavy vehicle, as they all exceed a $10,000 \mathrm{~kg}$ mass once all of the appropriate margins and reserves are accounted for. However, both the 9-m obscured and 8-m unobscured apertures can still be packaged into a 5-m-class fairing, offering some risk mitigation against LUVOIRA's requirement for the larger fairing of the SLS Block 2 vehicle.

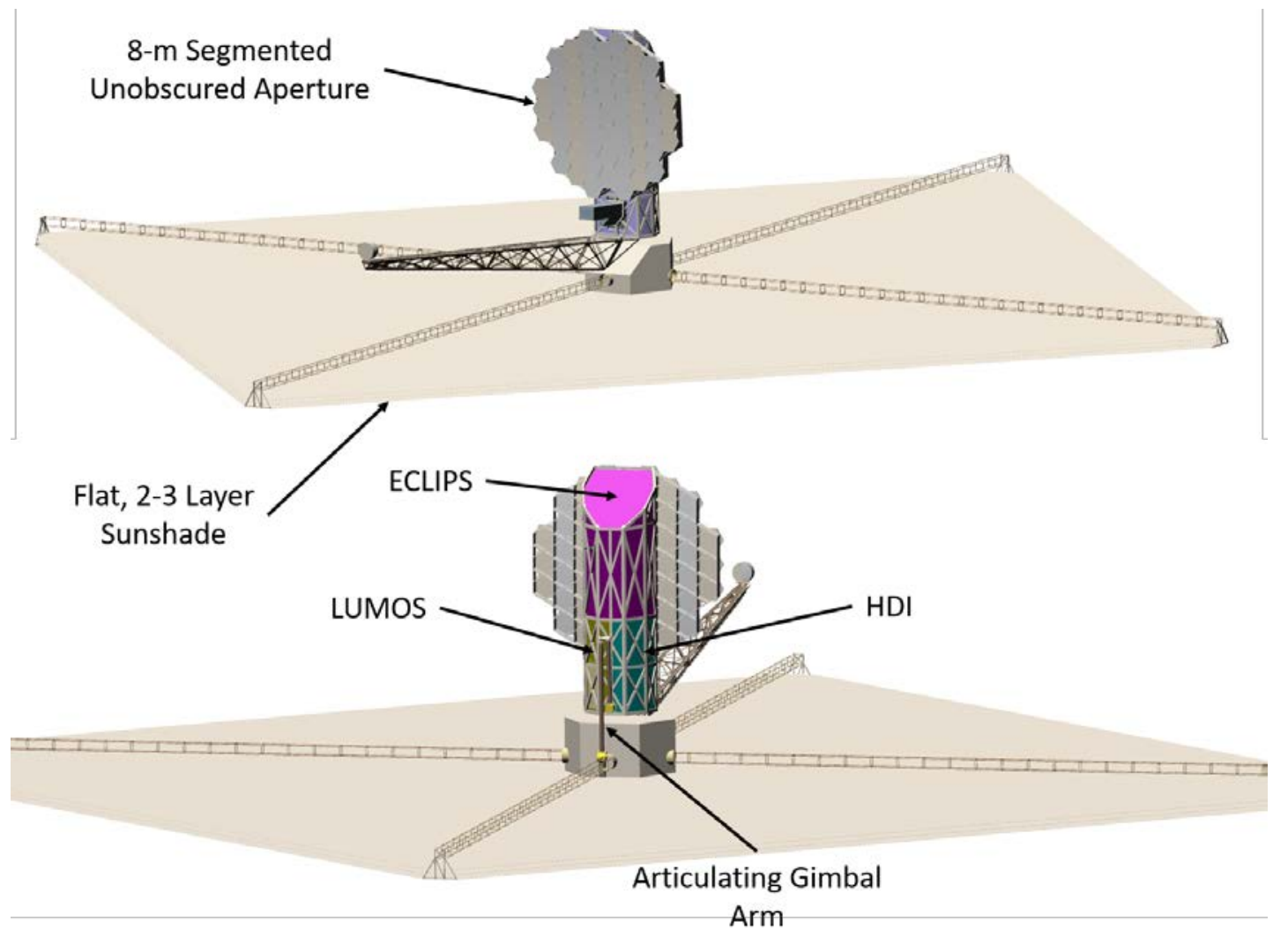

Figure 6 - The LUVOIR-B observatory concept.

Figure 6 shows the LUVOIR-B observatory concept. The primary mirror is composed of 55 segments that are $0.955-\mathrm{m}$ flat-to-flat. The OTA uses a three-mirror anastigmat design, including a fourth fast-steering mirror at the real exit pupil, 
similar to LUVOIR-A (for more details on the optical design of both architectures see Ref. 13). However, due to volume limitations, only three instrument bays are being baselined for LUVOIR-B. We note that while this concept shows the three instruments to be versions of ECLIPS, LUMOS, and HDI, the actual instrument suite would likely be competed and include contributions from international partners. Thus, even though Pollux is not explicitly shown on the LUVOIR-B concept, it could still be a candidate for a first-launch instrument.

A new method of stowing and deploying the secondary mirror had to be developed to enable the nearly 20-m separation between the primary mirror and secondary mirror, while keeping the primary mirror unobscured and the secondary mirror position stable. Figure 7 shows a sequence depicting this deployment concept. While this deployment concept departs significantly from the JWST-style deployment used on LUVOIR-A, we note that it leverages similar mechanisms as those on JWST: hinges, latches, and composite structures, and that all of the motions are single-axis, deterministic actuations. The resulting triangular truss provides a stable platform for positioning the secondary mirror and sending the optical beam to the aft-optics system.

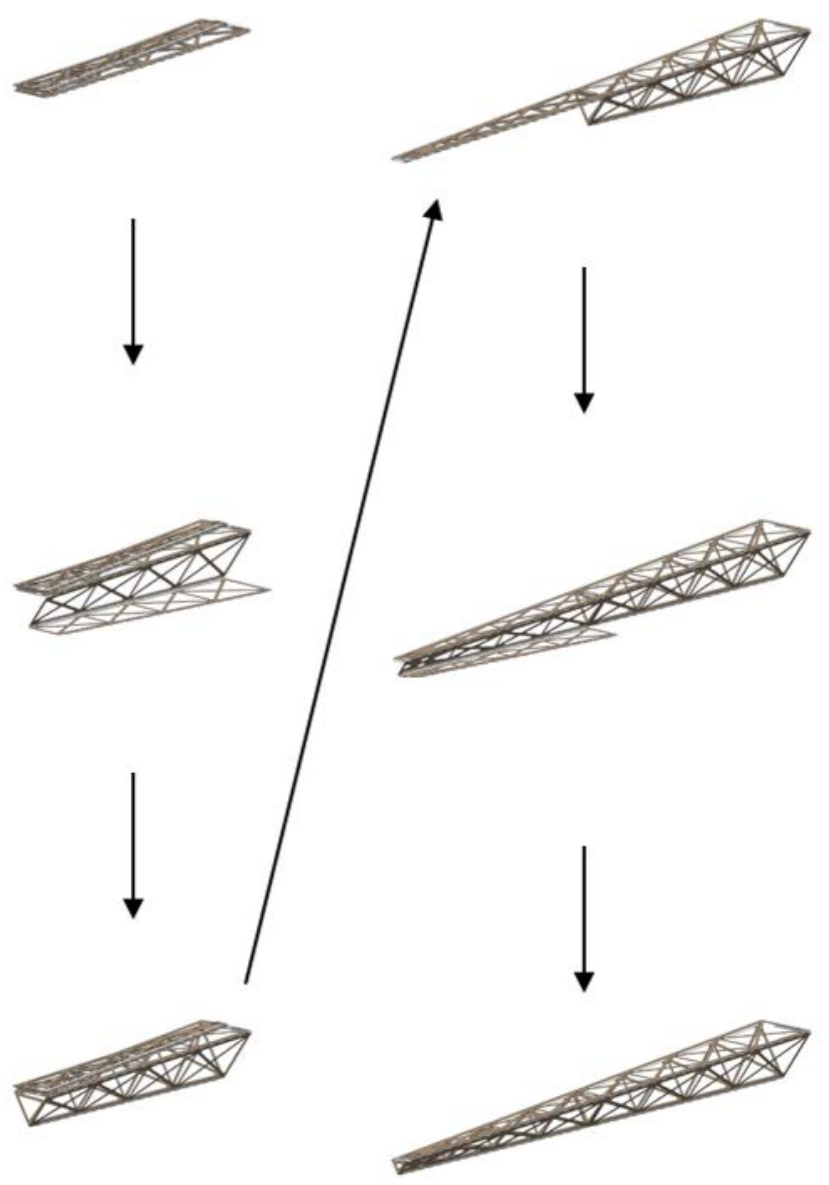

Figure 7 - Deployment sequence for the LUVOIR-B secondary mirror support structure. Staring in the upper left where the truss members are folded flat on top of each other, the middle-left image shows the first triangular section of the truss unfolding to create the first half of the support structure in the lower-left corner. The second length of the structure unfolds from there in the upper-right image. The middle-right triangular section then unfolds. Preloaded latches hold the entire truss structure stable in the lower right image.

Aside from these departures, the LUVOIR-B observatory concept shares many similarities with the LUVOIR-A concept. Both have a large, flat, two-to-three layer sunshade ${ }^{14}$ for thermal stability and to cold bias the observatory for active thermal control. Both observatories operate at $270 \mathrm{~K}$. Both use a multi-axis arm to articulate the payload relative to the spacecraft for access to the full anti-sun hemisphere, and both observatories are capable of tipping up to $40^{\circ}$ towards the 
sun to view inner-planets and comets, as well as execute time-sensitive exoplanet revisit observations. Both concepts also use a vibration isolation and precision point system (VIPPS) $)^{15,16,17}$ for dynamic isolation and fine payload pointing control.

\section{THE LUVOIR INSTRUMENTS}

\subsection{Extreme Coronagraph for Living Planetary Systems (ECLIPS)}

The ECLIPS instrument performs the high-contrast imaging and spectral characterization of exoplanets for LUVOIR. ECLIPS-A and ECLIPS-B are nearly identical in design. Both have three channels, split using dichroic elements so that each channel can be operated simultaneously. Table 2 summarizes the performance of each channel. Each channel uses two deformable mirrors (DMs) for focal plane wavefront control. Each channel also has an assortment of apodizing masks, focal plane masks, Lyot stops, and spectral filters to tune the channel's inner working angle, outer working angle, and spectral bandpass to the specific observation. Each channel also has a low-order / out-of-band wavefront sensor for maintaining wavefront stability during observations. The ECLIPS optical design, including the backend imaging and spectrograph instruments, is described in Ref. 18. Further details on the coronagraph mask designs and performance predictions are described in Ref. 19.

Table 2 - ECLIPS instrument channel specifications.

\begin{tabular}{|c|c|c|c|}
\hline Channel & Wavelength Range & Half Field-of-View & $\begin{array}{c}\text { Backend } \\
\text { Instrument }\end{array}$ \\
\hline UV & $200 \mathrm{~nm}-525 \mathrm{~nm}$ & $0.176 ”$ & $\begin{array}{c}\text { Imaging Camera } \\
\text { Only }\end{array}$ \\
\hline Visible & $515 \mathrm{~nm}-1030 \mathrm{~nm}$ & $0.450 ”$ & $\begin{array}{c}\text { Imaging Camera } \\
\text { or } \\
\mathrm{R}=140 \text { Integral } \\
\text { Field Spectrograph }\end{array}$ \\
\hline NIR & $1000 \mathrm{~nm}-2000 \mathrm{~nm}$ & $0.88 ”$ & $\begin{array}{c}\text { R=70 Integral Field } \\
\text { Spectrograph } \\
\text { Or } \\
\text { R=200 Point-source } \\
\text { Slit Spectrograph }\end{array}$ \\
\hline
\end{tabular}

One key difference between the ECLIPS-A and ECLIPS-B designs is the type of coronagraph that is baselined to be the primary mode. An apodized pupil Lyot coronagraph (APLC) works best with the LUVOIR-A obscured aperture, and thus most of the mask slots are dedicated to that type of coronagraph architecture. For LUVOIR-B's unobscured aperture, a vector vortex coronagraph provides superior inner working angle and throughput. Even so, both instruments will carry masks for the alternate design in order to reduce risk, as well as provide optimal performance for certain observations.

\subsection{High Definition Imager (HDI)}

HDI is the primary wide-field-of-view imaging instrument for LUVOIR. Both HDI-A and HDI-B have similar optical designs. Both consist of two channels: a UV-Visible (UVIS) channel covering wavelengths between $200 \mathrm{~nm}$ and 1000 $\mathrm{nm}$, and a NIR channel covering wavelengths between $800 \mathrm{~nm}$ and $2100 \mathrm{~nm}$. A channel selection mechanism determines which channel or channels can be used via one of five elements:

1. A reflective element that directs all of the light into the UVIS channel.

2. A transmissive element that directs all of the light into the NIR channel.

3. A 50/50 beamsplitter that allows both channels to be operated simultaneously over their full bandpasses, but at reduced throughput.

4. A dichroic that allows each channel to be operated at full throughput, but over reduced bandpass ( $400 \mathrm{~nm}-800 \mathrm{~nm}$ in the UVIS, $800 \mathrm{~nm}-1600 \mathrm{~nm}$ in the NIR).

5. A UV-optimized reflective element that maximizes the throughput in the blue-end of the spectrum in the UVIS channel. 
Both versions of HDI are Nyquist sampled $(1$ pixel $=\lambda / 2 \mathrm{D})$ at $500 \mathrm{~nm}$ in the UVIS channel and at $1000 \mathrm{~nm}$ in the NIR channel, and both versions of HDI have a $\sim 2 \times 3$ arcmin field-of-view. Thus, since the aperture diameters of LUVOIR-A and LUVOIR-B are different, so too are the detector and pixel counts in each version of the instrument. Table 3 summarizes the focal plane specifications for each instruments.

Table 3 - Summary of HDI focal plane specifications for each architecture.

\begin{tabular}{|c|c|c|c|c|c|c|c|c|}
\hline \multirow{2}{*}{ Instrument } & Channel & $\begin{array}{c}\text { Pixel } \\
\text { Size } \\
(\boldsymbol{\mu m})\end{array}$ & $\begin{array}{c}\text { Pixel } \\
\text { Size } \\
\mathbf{( m a s )}\end{array}$ & $\begin{array}{c}\text { Detector } \\
\text { Type }\end{array}$ & $\begin{array}{c}\text { Single } \\
\text { Detector } \\
\text { Format }\end{array}$ & $\begin{array}{c}\text { Array } \\
\text { Tiling }\end{array}$ & $\begin{array}{c}\text { Total } \\
\text { Number } \\
\text { of Pixels }\end{array}$ & $\begin{array}{c}\text { Actual } \\
\text { Field-of- } \\
\text { View } \\
\text { (arcmin) }\end{array}$ \\
\hline \multirow{2}{*}{ HDI-A } & UVIS & 6.5 & 3.44 & CMOS & $8 \mathrm{~K} \times 8 \mathrm{~K}$ & $6 \times 4$ & $1.6 \mathrm{Gpix}$ & $3.20 \times 1.93$ \\
\cline { 2 - 9 } & NIR & 10 & 6.88 & HgCdTe & $4 \mathrm{~K} \times 4 \mathrm{~K}$ & $6 \times 4$ & $402 \mathrm{Mpix}$ & $3.31 \times 1.95$ \\
\hline \multirow{2}{*}{ HDI-B } & UVIS & 6.5 & 6.45 & CMOS & $8 \mathrm{~K} \times 8 \mathrm{~K}$ & $3 \times 2$ & $403 \mathrm{Mpix}$ & $2.71 \times 1.80$ \\
\cline { 2 - 9 } & NIR & 10 & 12.90 & HgCdTe & $4 \mathrm{~K} \times 4 \mathrm{~K}$ & $3 \times 2$ & $101 \mathrm{Mpix}$ & $2.73 \times 1.81$ \\
\hline
\end{tabular}

Figure 8 shows a ray trace of the HDI-B optical design behind the 8-m off-axis primary mirror, with key elements labelled. For more details about the HDI instrument, see Ref. 6.

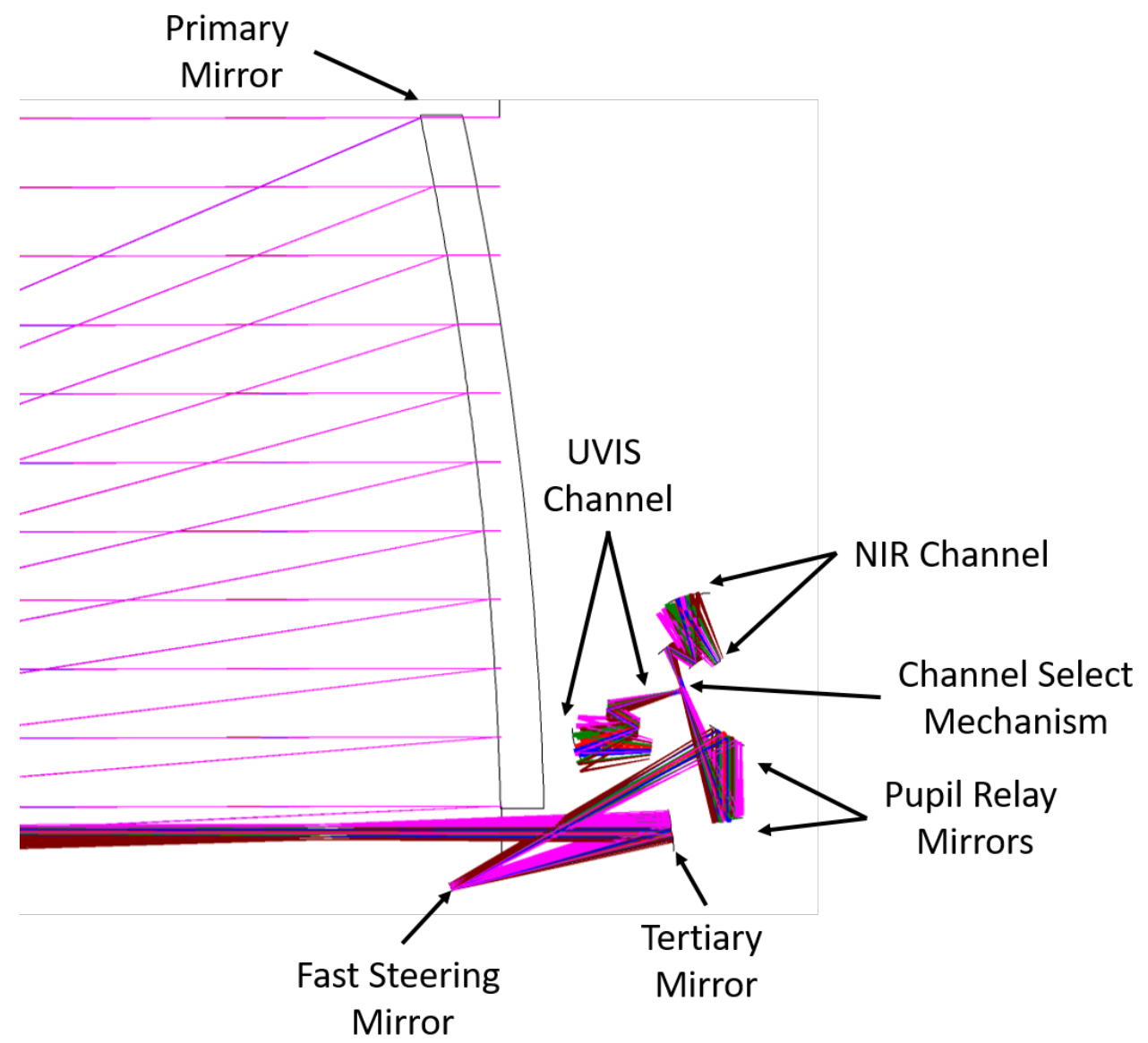

Figure 8 - Ray trace of the HDI-B instrument behind the 8-m unobscured LUVOIR-B telescope. The primary mirror, tertiary mirror, and fast-steering mirror are part of the OTA (the secondary mirror is off the page to the left), while all other elements are part of the HDI instrument. 


\subsection{LUVOIR UV Multi-object Spectrograph (LUMOS)}

LUMOS is the primary far-UV instrument on LUVOIR and consists of two primary operating modes: a far-UV to visible multi-object spectrograph and a far-UV imager. On LUMOS-A, these two functions are achieved with completely separate channels that have independent fields-of-view. On LUMOS-B, the two functions are combined into a single channel and share the same field-of-view.

The multi-object spectrograph (MOS) function uses a microshutter array at the OTA focal plane to select which objects will be observed. A selection of gratings provides varying resolutions for different bandwidths, and the dispersed scene imaged onto either a far-UV focal plane consisting of microchannel plate detectors, or a near-UV focal plane consisting of delta-doped CMOS detectors.

The far-UV imaging function uses reflective filters in series to define the optical bandpass and microchannel plate detectors to image the scene. A set of neutral density filters are also included for bright-object protection. On LUMOS-B, the imaging is done through the microshutter array, since both channels share the same field-of-view.

Table 4 summarizes key specifications of the LUMOS instruments, and Figure 9 shows a ray trace of the LUMOS-B optical design with key elements labelled. For more details about the LUMOS instrument, see Refs. 6 and 20.

Table 4 - LUMOS instrument capability summary.

\begin{tabular}{|c|c|c|c|c|c|}
\hline Instrument & Channel & FOV & $\begin{array}{c}\text { Wavelength } \\
\text { Range }\end{array}$ & $\begin{array}{c}\text { Angular } \\
\text { Resolution }\end{array}$ & $\begin{array}{c}\text { Spectral } \\
\text { Resolution }\end{array}$ \\
\hline \multirow{2}{*}{ LUMOS-A } & MOS & $2 \times 2$ & $100 \mathrm{~nm}-400 \mathrm{~nm}$ & $<24$ mas & $\begin{array}{c}13,000- \\
56,000\end{array}$ \\
\cline { 2 - 6 } & Imager & $2 \times 2$ & $100 \mathrm{~nm}-200 \mathrm{~nm}$ & 13 mas & N/A \\
\hline LUMOS-B & MOS / Imager & $2 \times 2$ & $\begin{array}{c}100 \mathrm{~nm}-1000 \\
\mathrm{~nm}(\mathrm{MOS} \text { mode) } \\
100 \mathrm{~nm}-200 \mathrm{~nm} \\
\text { (Imaging Mode) }\end{array}$ & $\sim 30$ mas & $\begin{array}{c}\sim 10,000- \\
60,000\end{array}$ \\
\hline
\end{tabular}

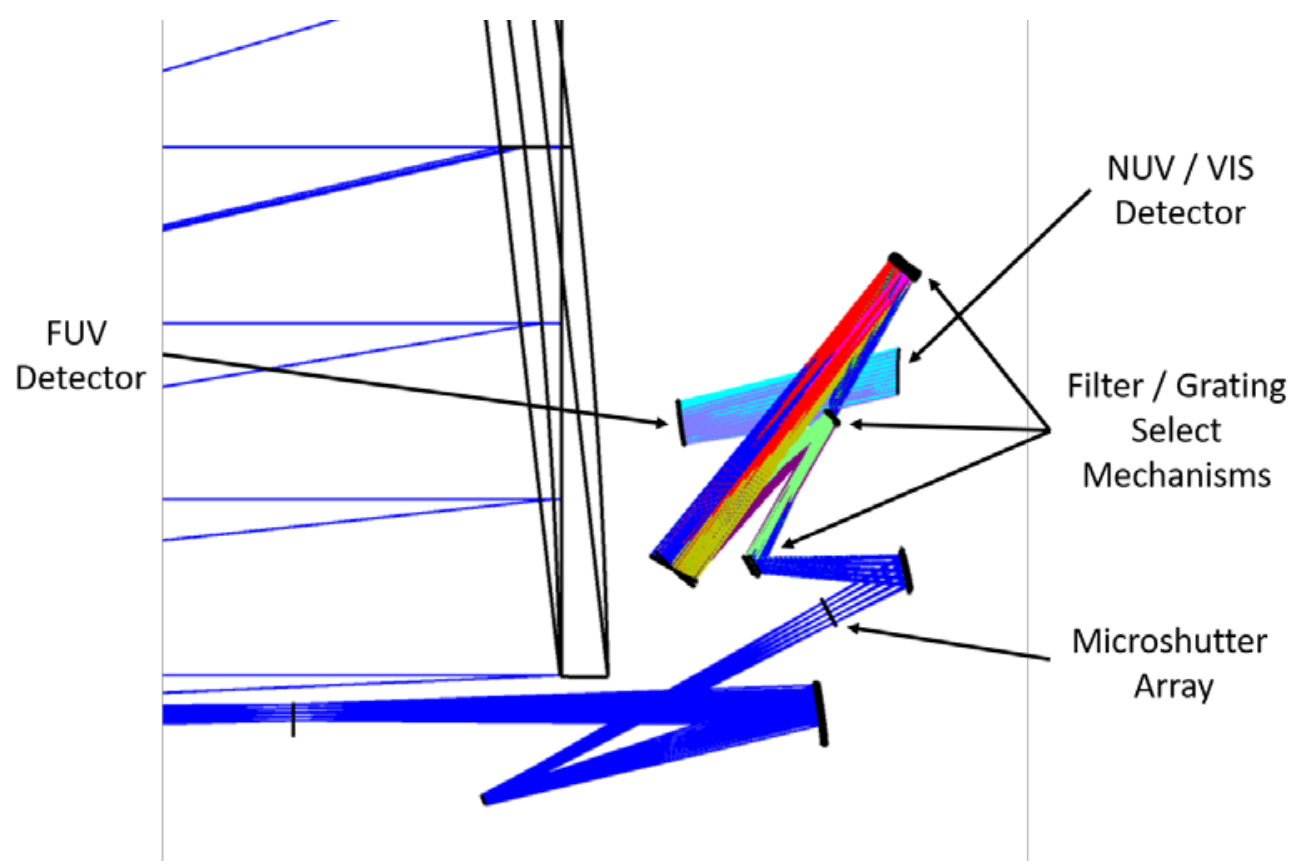

Figure 9 - Ray trace of the LUMOS-B instrument behind the 8-m unobscured LUVOIR-B telescope. The microshutter array is located at the OTA focal plane, and the three filter / grating select mechanisms determine which mode the instrument is operated in, and which detector is illuminated. 


\section{FUTURE WORK}

Now that both mission concepts have been defined, the LUVOIR Study Team will continue to develop them for submission to the 2020 Decadal Survey in Astrophysics and Astronomy. At least one of these concepts will be brought to a Concept Maturity Level (CML) of $4^{21}$, such that it can be submitted to an independent cost estimation by an external non-advocate party in the spring of 2019, as well as by the Decadal Committee in late 2019 or early 2020. The LUVOIR STDT is currently evaluating which of the two concepts to develop to CML4, however both concepts will be presented to the Decadal Committee for consideration.

The Study Team's CML4 deliverable is due to NASA HQ in January of 2019, and will include materials such as technical resource budgets, master equipment lists, a technology development plan, a science traceability matrix, an implementation schedule, and a definition of risk drivers, etc. After the independent cost estimation process, the Study Team will have a chance to iterate the design one last time to incorporate any feedback before submitting the final report to the National Academies in July of 2019.

\section{REFERENCES}

${ }^{1}$ P. Hertz, "Charter for the Mission Concept Study Science and Technology Definition Teams,” NASA, 28 December 2015, https://science.nasa.gov/astrophysics/2020-decadal-survey-planning/ (accessed 29 August 2017).

${ }^{2}$ See: https://ads.gsfc.nasa.gov/luvoir/

${ }^{3}$ See: https://www.jpl.nasa.gov/habex/

${ }^{4}$ See: https://asd.gsfc.nasa.gov/firs/

${ }^{5}$ See: https://wwwastro.msfc.nasa.gov/lynx/

6 “The LUVOIR Study Interim Report”, in review, 2018. Will be posted at https://asd.gsfc.nasa.gov/luvoir/ when available.

${ }^{7}$ See: https://asd.gsfc.nasa.gov/luvoir/tools/

${ }^{8}$ E.R. Muslimov, et al., "POLLUX: a UV spectropolarimeter for the future LUVOIR space telescope," Proc. SPIE, 10699, 10699-05, 2018.

${ }^{9}$ J.C. Bouret, et al., "The case for POLLUX: a high-resolution UV spectropolarimeter onboard LUVOIR,” Proc. SPIE, 10699, 10699-118, 2018.

${ }^{10}$ C.C. Stark, et al., "Lower Limits on Aperture Size for an ExoEarth Detecting Coronagraphic Mission,” Ap. J., 808:149, 2015.

${ }^{11}$ J. Wang, et al., "Observing Exoplanets with High Dispersion Coronagraphy II: Demonstration of an Active Singlemode Fiber Injection Unit,” Ap. J., 838:2, 2017.

${ }^{12}$ M.R. Bolcar, et al., "The Large UV/Optical/Infrared Surveyor (LUVOIR): Decadal Mission concept design update," Proc. SPIE, 10398, 1039809, 2017.

${ }^{13}$ G.J. West, et al., "Optical design and status of the large ultra-violet optical infrared surveyor (LUVOIR),” Proc. SPIE, 10698, 10698-138, 2018.

${ }^{14}$ J. Arenberg, et al., "LUVOIR thermal architecture,” Proc. SPIE, 10698, 10698-39, 2018.

${ }^{15}$ L.W. Sacks, et al., "Preliminary jitter stability results for the large UV/optical/infrared (LUVOIR) surveyor concept using a non-contact vibration isolation and precision pointing system,” Proc. SPIE, 10698, 10698-141, 2018.

${ }^{16}$ L.D. Dewell, et al., "Telescope line-of-sight slew control and agility with non-contact vibration isolation for the large ultraviolet/optical/infrared (LUVOIR) surveyor,” Proc. SPIE, 10698, 10698-137, 2018.

${ }^{17}$ L.D. Dewell, et al., "Dynamic stability with the disturbance-free payload architecture as applied to the Large UV/Optical/Infrared (LUVOIR) Mission,” Proc. SPIE, 10398, 103980B, 2017.

${ }^{18} \mathrm{Q}$. Gong, et al., "Optical design of exo-planet coronagraph, integral field spectrograph, and high resolution spectrometer for LUVOIR study,” Proc. SPIE, 10698, 10698-37, 2018.

${ }^{19}$ L. Pueyo, et al., “The LUVOIR coronagraph instrument,” Proc. SPIE, 10698, 10698-34, 2018.

${ }^{20}$ K. France, et al., "The LUVOIR Ultraviolet Multi-object Spectrograph (LUMOS): instrument definition and design," Proc. SPIE, 10397, 1039713, 2017.

${ }^{21}$ R. Wessen, et al., “Space Mission Concept Development Using Concept Maturity Levels,” AIAA SPACE 2013

Conference and Exposition, AIAA SPACE Forum, (AIAA 2013-5454). 